\title{
Irradiation tests of the pixel front-end readout electronics for the ALICE experiment at LHC
}

\author{
A. Badalà, R. Barbera, A. Palmeri, G. S. Pappalardo, F. Riggi* \\ INFN Catania and University of Catania, Italy \\ S. Di Liberto, F.Meddi ${ }^{* *}$, A.Cavagnoli \\ INFN Roma and University La Sapienza, Roma, Italy \\ M.Morando, F.Scarlassara, G.Segato, F.Soramel \\ INFN Padova and University of Padova, Italy \\ L.Vannucci \\ INFN Legnaro, Italy
}

for the ALICE Collaboration

(June 26, 2000)

\begin{abstract}
The problem of radiation damage for the electronics of the pixel detectors in the Inner-Tracking-System of the ALICE experiment is discussed. Simulations allowed to estimate cumulated doses and particle fluences during a ten years operational period. Several irradiation tests have been carried out on the various prototypes of the readout chips. The results obtained so far point out that the recent prototypes will retain their functionality up to doses and neutron fluences well above those expected in ALICE.
\end{abstract}


* Corresponding Author:

Francesco Riggi, Department of Physics and Astronomy,

University of Catania, I95129 Catania, Italy

Fax ++39 095 383023, Tel. ++39 095 7195261, e-mail: Franco.Riggi@ct.infn.it

** Now at CERN-EP, Geneve, CH

PACS number: 29.40.Gx, 07.50.-e, 07.89.+b

Keywords: Pixel; Detector; Silicon; Radiation Damage; Microelectronics 


\section{INTRODUCTION}

An important part of the design of the Inner-Tracking-System (ITS) [1] of the ALICE experiment at the CERN LHC has been the implementation of radiation tolerant technologies for the electronic circuitry located close to the interaction region. Such problem is of special concern for the electronics of the pixel detectors, which constitute the innermost two layers in the ALICE ITS. These layers are essential for the reconstruction of the primary vertex and for the measurement of the impact parameter of the secondary tracks originating from the decay of strange, charm and beauty particles. Such requirements can only be fulfilled by detectors which couple high precision and granularity, in order to operate near the collision vertex, where the track density may exceed 50 tracks $/ \mathrm{cm}^{2}$. The pixel detectors must survive to an expected overall dose in the order of $200 \mathrm{krad}$ in ten years [2,3]. This has demanded an effort in the electronic design which led to a sensible upgrading of the hardness with respect to the first readout chips of the Omega family, still in use in experiments at SPS. This effort from the Microelectronics Group at CERN has been accompanied by a series of irradiation tests carried out in several laboratories with different probes.

Sect.II discusses the radiation environment envisaged for the ITS during a realistic working scenario of the ALICE experiment. The development of the pixel readout electronics is shortly presented in Sect.III, while some results concerning experimental tests of the radiation hardness for the readout electronics are reported in Sect.IV. Finally, some conclusions are drawn in Sect.V.

\section{THE RADIATION ENVIRONMENT FOR THE PIXEL LAYERS IN THE ALICE ITS}

At an energy around $6 \mathrm{~A} \mathrm{TeV}$, planned for the heavy-ion LHC program, a large particle multiplicity is predicted by the current theoretical models, reaching values around $10^{5}$ for a central $\mathrm{Pb}-\mathrm{Pb}$ collision. Full simulations of the interaction of the primary and secondary 
particles generated by a heavy-ion collision with the overall structure of the ALICE detector have been carried out [3]. This allowed to evaluate the cumulated dose and particle fluence on the ITS detectors. Most of the simulations made use of the event generator program HIJING (Heavy Ion Jet INteraction Generator), version 1.35 [4]. This code was used to generate central, peripheral and intermediate impact parameter collisions, with a proper mixing of the events to simulate the real collisions. Primary and secondary particles were tracked using a GEANT package describing as close as possible the geometry and materials planned for the ALICE detectors.

In order to scale from the expected dose per event, evaluated for individual collision classes, to the overall cumulated dose, a reasonable scenario during ten years data taking can be found in the ALICE Technical Proposal (see Table I), which includes proper sharing of beam time between protons, $\mathrm{Ca}$ and $\mathrm{Pb}$ ions. A summary of the cumulated dose expected in the individual ITS layers in an operational period of ten years is reported in Table II and Fig. 1. The use of different event generators with the largest value of the pseudorapidity distribution $\left(\mathrm{dN}_{c h} / \mathrm{d} \eta=8000\right)$ could increase the overall dose up to $200 \mathrm{krad}$ for the innermost pixel layer.

Neutron fluences have been estimated by FLUKA simulations, which give values around $3 \times 10^{11} \mathrm{n} / \mathrm{cm}^{2}$ (energy-integrated) for the pixel layers (see Table II). Recent simulations [5] also provide the contribution to the neutron fluence due to the front absorber and the energy distribution of the secondary neutrons, with a maximum yield in the range $100 \mathrm{keV}-1 \mathrm{MeV}$.

\section{DEVELOPMENT OF THE PIXEL READOUT ELECTRONICS}

The first series of the pixel front-end readout chips (Omega2 and Omega3) are employed in the WA97 and NA57 experiments at CERN SPS [6]. While satisfying many of the requirements for the ALICE experiment, they were rather sensitive to radiation. The Omega3 front-end chip is a matrix of $16 \times 127$ active readout cells $\left(50 \times 500 \mu \mathrm{m}^{2}\right)$, manifactured in $1 \mu \mathrm{m}$ SACMOS process, giving the same component density of a $0.6 \mu \mathrm{m}$ standard CMOS 
process. Each front-end cell includes a preamplifier, a shaper filter, a discriminator, a delay line and the readout logic. A mask flip-flop allows the cell to be disabled when it is too noisy or defective (Fig. 2).

Further requirements in terms of the electronic circuitry needed for the ALICE experiment demanded a process with both a high component density and a good tolerance to radiation.

As a result of an extensive R\&D work [7] done by the Microelectronics Group at CERN, two prototypes (ALICE1test and ALICE2test) have been developed. The first is a small test chip (65 x 2 cells, each measuring $50 \times 420 \mu \mathrm{m}^{2}$ ), developed in $0.5 \mu \mathrm{m}$ CMOS technology, with the use of NMOS devices in enclosed geometry and guardrings. The use of such techniques enhanced the radiation tolerance by a factor of 30-50 with respect to the Omega 3 series (see following Section). Such performance is adequate for the requirement of the ALICE experiment. However, the need for an even larger component density brought to the choice of a deeper submicron technology $(0.25 \mu \mathrm{m})$ for the design of the ALICE2test chip. Similar to the previous chip, ALICE2test is a matrix of $65 \times 2$ cells, containing this time about 50000 transistors. The design of this second test chip also enabled to incorporate some additional features required for the final ALICE chip, such as an individual pixel threshold adjust and a delay line based on the use of a 8 bit counter.

\section{EXPERIMENTAL TESTS OF THE RADIATION DAMAGE FOR THE PIXEL ELECTRONICS}

Systematic studies concerning the radiation tolerance of front-end chips of the Omega series were carried out by X-rays [1,8], and using a ${ }^{60} \mathrm{Co}$ gamma source [9], $15 \mathrm{MeV}$ electrons $[10]$ and a proton beam [9]. These studies all demonstrated that these chips cannot survive to doses significantly higher than $30 \mathrm{krad}$. As a result, a common protocol for the irradiation of the submicron technology prototypes was established. With the advent of the new prototypes, built in radiation-tolerant technology, additional irradiation tests were 
performed with various sources [1]. First of all, X-ray irradiations were carried out at CERN under the same conditions as for the individual transistors [8]. The result showed on a full circuit scale the effectiveness of enclosed NMOS devices and guardrings to prevent radiationinduced leakage. The ALICE1test chip was seen to degrade significantly only after $600 \mathrm{krad}$. Irradiations by $\gamma$-rays from ${ }^{60} \mathrm{Co}$ at the Istituto Superiore di Sanità (Rome) were then carried out at a dose rate of $610 \mathrm{rad} / \mathrm{min}$, with a uniformity within $5 \%$ on the chip. The evolution of the supply currents (analog and digital), as well as the threshold and its dispersion were studied as a function of the absorbed dose. The results pointed out a functionality of the chip up to doses around 1 Mrad, where a a degradation of the performance, including the number of responding cells, was seen. Partial recovery after a few days from irradiation was observed in the discriminator threshold, due to annealing effects. The tolerance to charged hadronic particles was also investigated, by the use of $6.5 \mathrm{MeV}$ protons at the $\mathrm{CN}$ accelerator of the Legnaro National Laboratory, with a dose rate of $12 \mathrm{krad} / \mathrm{min}$. Even in this case, serious degradation takes place only after $1 \mathrm{Mrad}$, with partial recover during the annealing phase. In another test [1] carried out at CERN with low energy electrons derived from the NA50 experiment, the chip was seen to survive up to a dose of $1.7 \mathrm{Mrad}$.

Even though it is extremely difficult to compare results concerning the damage caused by different radiation sources, a qualitative agreement between all these results was observed, which allows to establish a radiation tolerance for such prototype, built in $0.5 \mu \mathrm{m}$ CMOS technology, up to doses in the order of $1 \mathrm{Mrad}(0.6-1.7 \mathrm{Mrad})$, which is a factor about 30-50 with respect to the Omega3 chip.

Similarly to the previous prototype, the first measurements concerning the radiation tolerance of the ALICE2test chip were done by X-rays with a peak energy of $10 \mathrm{KeV}[1,8]$. The chip was seen to be fully operational up to $30 \mathrm{Mrad}$, with a slight change of the pixel threshold and noise after 10 Mrad. Even in such case, partial recovery due to annealing was observed. Further tests were carried out with ${ }^{60} \mathrm{Co} \gamma$-rays up to $26 \mathrm{Mrad}$, observing a slight increase of the analog current (Fig. 3, top), and with $6.5 \mathrm{MeV}$ protons with doses of 9, 19 and $48 \mathrm{Mrad}$ (Fig. 3, bottom), where the same trend was observed. The chip ceased to work 
at $48 \mathrm{Mrad}$. High energy protons were also used at the CERN SPS machine, with a fluence of about $9 \times 10^{14}$ protons $/ \mathrm{cm}^{2}$ over a $12 \mathrm{~h}$ period. A recent test [11] was also done with 14 MeV neutrons at the Frascati neutron generator using the $\mathrm{T}(\mathrm{d}, \mathrm{n}) \alpha$ reaction. A fluence up to $1.3 \times 10^{12} \mathrm{n} / \mathrm{cm}^{2}$ was used, which corresponds roughly to $2.3 \times 10^{12} 1 \mathrm{MeV}$-equivalent neutrons $/ \mathrm{cm}^{2}$. The chip was powered as usual during all the irradiations, monitoring both the analog and digital currents (Fig. 4) which were seen to remain constant within $0.5 \%$. A detailed electrical characterization of the chip prior and after the irradiation was also undertaken, checking the shape of the signals from the preamplifier and from the shaper, the linearity in the gain and the efficiency response. No degradation effect was seen, up to this neutron fluence, which is approximately one order of magnitude higher than expected in ten years ALICE scenario. For the sake of completeness, a summary of the irradiation tests which have been carried out so far on the two prototypes, is reported in Table III.

\section{SUMMARY AND CONCLUSION}

An extensive work has been carried out to implement radiation tolerant techniques in the pixel front-end electronics for the ALICE experiment in order to reach the required hardness for the full operational period of the experiment. Several prototypes of the readout chips have been tested in various laboratories and employing very different probes, which include $\mathrm{X}$-rays, ${ }^{60} \mathrm{Co} \gamma$-rays, low and high energy protons, electrons and neutrons. Even though it is not easy to standardize a comparison between different sources of radiation, a common behaviour has been obtained by different irradiations. For the first prototype, built in 0.5 $\mu \mathrm{m}$ technology, a tolerance around 1 Mrad has been achieved, whereas the second may be considered as full operational up to doses of the order of $10 \mathrm{Mrad}$. This result is well in excess for the requirements of the ALICE experiment, discussed in Sect.II, and allows to conclude that the R\&D work, as far as the radiation tolerance is concerned, is now complete. We would like to stress that the conditions used in such irradiations are worser than the expected scenario during the ALICE run, because only a few months of exposure per year 
are planned, allowing annealing effects during the rest of each operational year.

\section{ACKNOWLEDGMENTS}

Thanks are due to the Microelectronics Group at CERN, where the R\&D work concerning the radiation tolerance of the pixel electronics has been carried out, and to A.Morsch for his FLUKA simulations of the neutron flux in the ITS. We are also indebted to F.Librizzi for his help during the irradiation with neutrons. 


\section{REFERENCES}

[1] The ALICE Collaboration, Inner-Tracking-System Technical Design Report, Report CERN/LHCC 99-12(1999).

[2] F.Meddi and M.Morando, Nucl. Instr. and Methods A395(1997)416.

[3] A.Badalà et al., Internal Note ALICE/99-01.

[4] X.N.Wang and M.Gyulassy, Phys. Rev. D44(1991)3501.

[5] A.Morsch, private communication; see also information at the following Web address: http://Alisoft.cern.ch/offline/

[6] F.Antinori et al., J.Physics G:Nucl.Part. Phys. 25(1999)473.

[7] W.Snoyes et al., Nucl. Instruments and Methods A439(2000)282.

[8] W.Snoyes, Proceedings of the Fourth Workshop on Electronics for LHC experiments, Report CERN/LHCC/98-36, p.114-118.

[9] S.Di Liberto et al., Internal Note ALICE/98-43.

[10] A.Badalà et al., Internal Note ALICE/99-31.

[11] A.Badalà et al., Internal Note ALICE/2000, in preparation. 


\section{FIGURES}

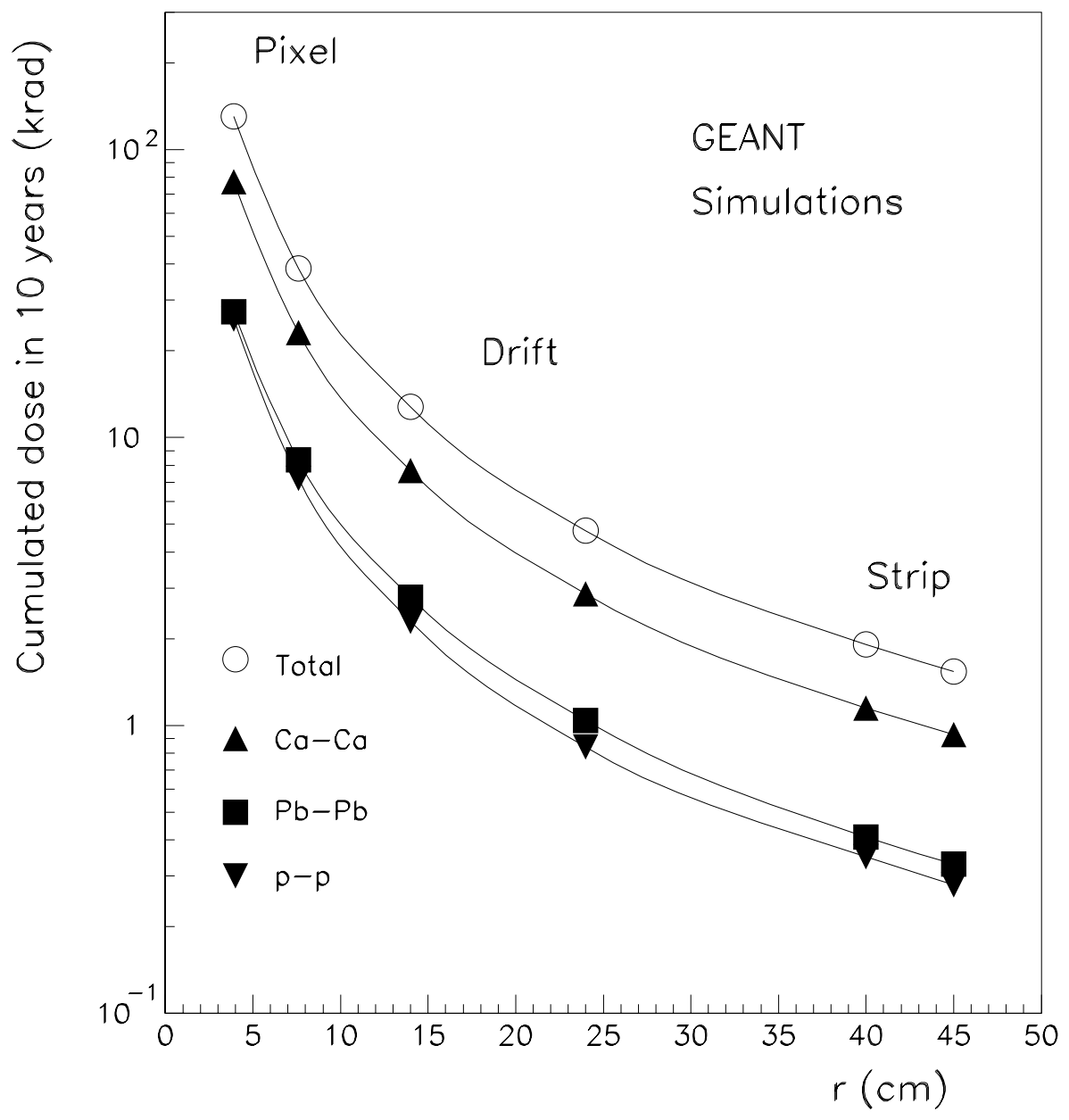

FIG. 1. Cumulated dose in the ITS layers during a running period of ten years, together with the contribution from $\mathrm{pp}, \mathrm{CaCa}$ and $\mathrm{PbPb}$ collisions. 


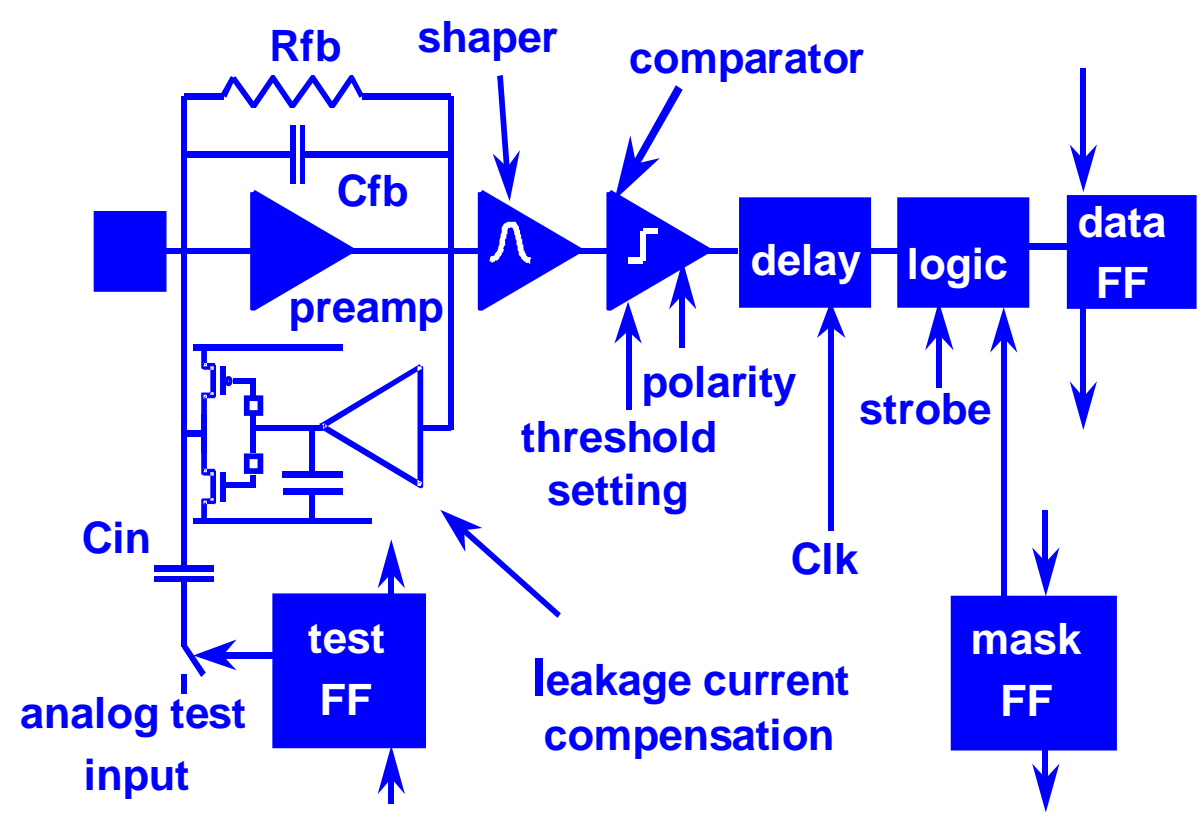

FIG. 2. Block diagram of a cell in the ALICE2test pixel readout chip. 


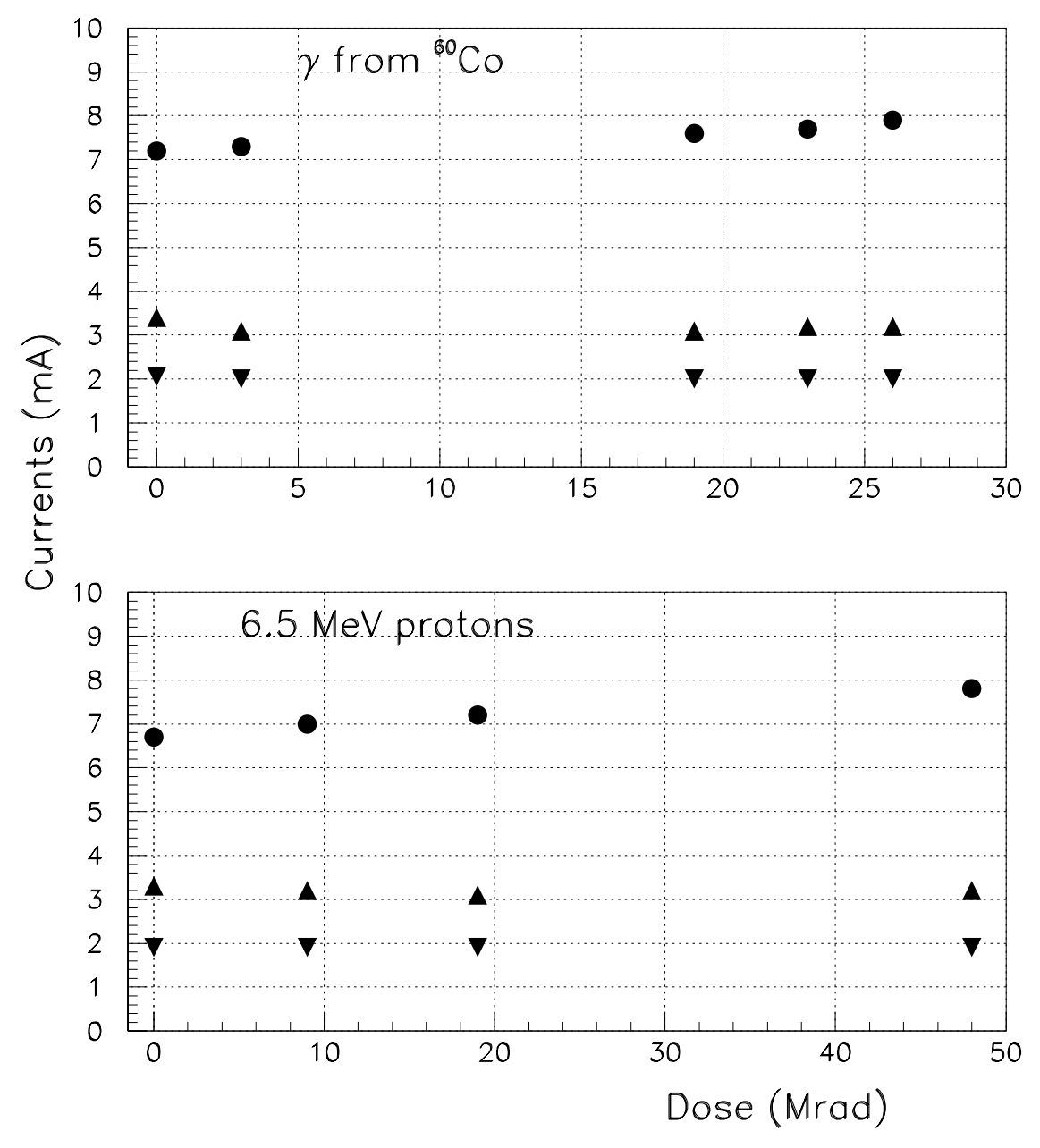

FIG. 3. Behaviour of the analog (dots) and of the minimum and maximum digital (triangles) supply currents for the ALICE2test chip during irradiation with $\gamma$-rays from ${ }^{60} \mathrm{Co}$ and $6.5 \mathrm{MeV}$ protons. 


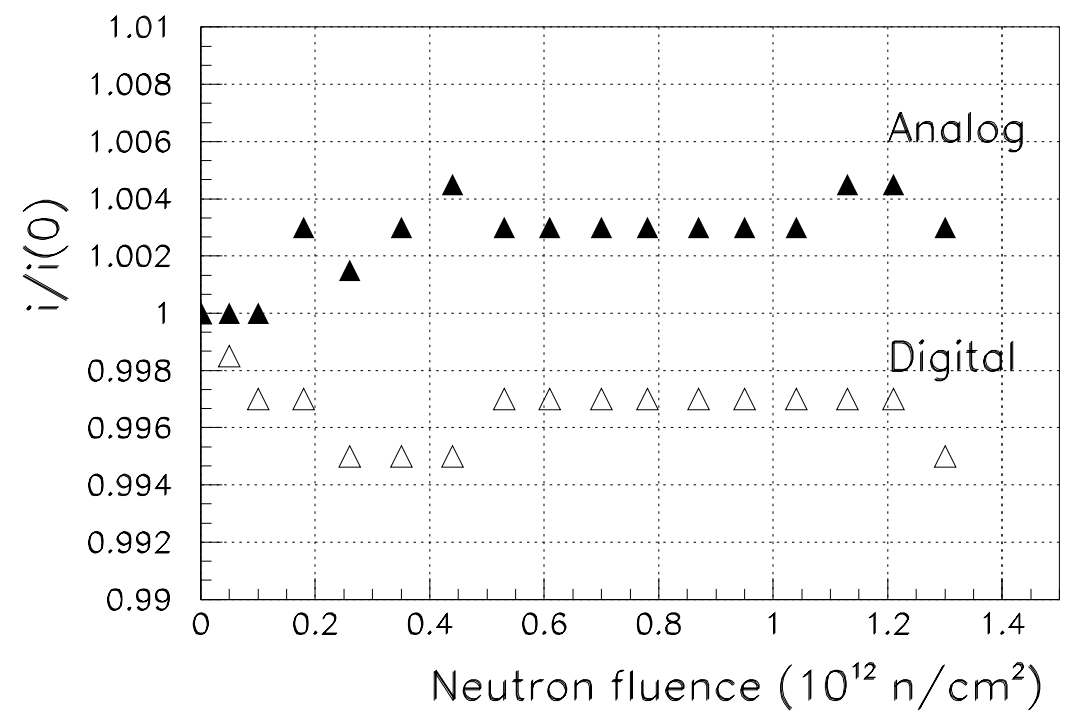

FIG. 4. Variation of the digital (open symbols) and analog (filled symbols) currents in the ALICE2test chip as a function of the neutron fluence. $14 \mathrm{MeV}$ neutrons have been employed. Fluctuations are of the same order as the instrumental sensitivity. 


\section{TABLES}

TABLE I. ALICE scenario for an overall working period of ten years.

\begin{tabular}{ccccc}
\hline \hline Parameter & $\mathrm{pp}$ & $\mathrm{Ca}-\mathrm{Ca}$ low L & $\mathrm{Ca}-\mathrm{Ca}$ high L & $\mathrm{Pb}-\mathrm{Pb}$ \\
\hline $\mathrm{L}\left(\mathrm{cm}^{-2} \mathrm{~s}^{-1}\right)$ & $10^{30}$ & $3 \times 10^{27}$ & $10^{29}$ & $10^{27}$ \\
Running time (s) & $10^{8}$ & $2.5 \times 10^{6}$ & $2.5 \times 10^{6}$ & $5 \times 10^{6}$ \\
Rate $\left(\mathrm{s}^{-1}\right)$ & $10^{5}$ & $8 \times 10^{3}$ & $3 \times 10^{5}$ & $8 \times 10^{3}$ \\
No. of events & $10^{13}$ & $2 \times 10^{10}$ & $7.5 \times 10^{11}$ & $4 \times 10^{10}$ \\
\hline \hline
\end{tabular}


TABLE II. Overall cumulated dose (krad) and neutron fluence in ten years for the individual ITS layers.

\begin{tabular}{cccccc}
\hline \hline Layer & $\mathrm{pp}$ & $\mathrm{CaCa}$ & $\mathrm{PbPb}$ & Total & neutron fluence $\left(10^{11} / \mathrm{cm}^{2}\right)$ \\
\hline 1 & 26 & 77 & 27 & 130 & 3.2 \\
2 & 7 & 23 & 8 & 39 & 3.1 \\
3 & 2.3 & 7.7 & 2.8 & 13 & 3.5 \\
4 & 0.8 & 2.9 & 1.0 & 4.7 & 3.3 \\
5 & 0.4 & 1.2 & 0.4 & 1.9 & 3.7 \\
6 & 0.3 & 0.9 & 0.3 & 1.5 & 3.3 \\
\hline \hline
\end{tabular}


TABLE III. Summary of irradiation tests carried out on the prototypes of the ALICE front-end pixel readout chips.

\begin{tabular}{cccc}
\hline \hline Source of radiation & Prototype & Max dose/particle fluence & Ref. \\
\hline $10 \mathrm{KeV}$ X-rays & ALICE1test & $800 \mathrm{krad}$ & {$[8]$} \\
${ }^{60} \mathrm{Co} \gamma$-rays & ALICE1test & $1.5 \mathrm{Mrad}$ & {$[1]$} \\
$1 \mathrm{MeV}$ electrons & ALICE1test & $2.6 \mathrm{Mrad}$ & {$[1]$} \\
$6.5 \mathrm{MeV}$ protons & ALICE1test & $1.05 \mathrm{Mrad}$ & {$[1]$} \\
$10 \mathrm{KeV}$ X-rays & ALICE2test & $30 \mathrm{Mrad}$ & {$[8]$} \\
${ }^{60} \mathrm{Co} \gamma$-rays & ALICE2test & $26 \mathrm{Mrad}$ & {$[1]$} \\
High energy protons & ALICE2test & $9 \times 10^{14} \mathrm{p} / \mathrm{cm}^{2}$ & {$[1]$} \\
$6.5 \mathrm{MeV}$ protons & ALICE2test & $48 \mathrm{Mrad}$ & {$[1]$} \\
$14 \mathrm{MeV}$ neutrons & ALICE2test & $1.3 \times 10^{12} \mathrm{n} / \mathrm{cm}^{2}$ & {$[11]$} \\
\hline \hline
\end{tabular}

